\title{
Epidemiology of Hydatidiform Moles in a Tertiary Hospital in Thailand over Two Decades: Impact of the National Health Policy
}

\author{
Varangkana Wairachpanich ${ }^{1}$, Sompop Limpongsanurak ${ }^{1}$, Ruangsak \\ Lertkhachonsuk ${ }^{2 *}$
}

\begin{abstract}
Background: The incidence of hydatidiform mole (HM) differs among regions but has declined significantly over time. In Thailand, the initiation of universal health coverage in $\mathbf{2 0 0 2}$ has resulted in a change of medical services countrywide. However, impacts of these policies on gestational trophoblastic disease (GTD) cases in Thailand have not been reported. This study aimed to find the incidence of hydatidiform mole (HM) in King Chulalongkorn Memorial Hospital (KCMH) from 1994-2013, comparing before and after the implementation of the universal coverage health policy. Materials and Methods: All cases of GTD in KCMH from 1994-2013 were reviewed from medical records. The incidence of HM, patient characteristics, treatment and remission rates were compared over two study decades between 1994-2003 and 2004-2013. Results: Hydatidiform mole cases decreased from 204 cases in the first decade to 111 cases in the seond decade. Overall incidence of HM was 1.70 per 1,000 deliveries. The incidence of $\mathrm{HM}$ in the first and second decades were 1.70 and 1.71 per 1,000 deliveries, respectively ( $p=0.65,95 \%$ CI 1.54-1.88). Referred cases of nonmolar gestational trophoblastic neoplasia (GTN) increased from $12(4.4 \%)$ to $23(14.4 \%, p<0.01)$. Vaginal bleeding was the most common presenting symptom which decreased from $89.4 \%$ to $79.6 \%(\mathrm{p}=0.02)$. Asymptomatic $\mathrm{HM}$ patients increased from $4.8 \%$ to $10.2 \%$ $(\mathrm{p}=\mathbf{0 . 0 7}$ ). Rate of postmolar GTN was $26 \%$. Conclusions: The number of HM cases in this study decreased over 2 decades but incidence was unchanged. Referral rates of malignant cases were more common after universal health coverage policy initiation. Classic clinical presentation was decreased significantly in the last decade.
\end{abstract}

Keywords: Hydatidiform mole - gestational trophoblastic disease - health care system - epidemiology

Asian Pac J Cancer Prev, 16 (18), 8321-8325

\section{Introduction}

Gestational trophoblastic disease (GTD) comprises a spectrum of diseases arising from abnormal proliferation of placental trophoblasts. It can be histologically categorized to benign hydatidiform mole (HM) and malignant gestational trophoblastic neoplasia (GTN). GTN can then be further classified into invasive mole, choriocarcinoma and placental-site trophoblastic tumor (PSTT)(Lurain and Brewer, 2010, Ngan et al., 2012).

$\mathrm{HM}$ is diagnosed by clinical symptoms such as abnormal vaginal bleeding, enlarged uterine size and hyperemesis gravidarum together with abnormal rising of B-hCG and vesicular ultrasonographic pattern (Berkowitz and Goldstein, 2012). However, asymptomatic patients increased due to early ultrasound screening (Ozalp et al., 2014). After treatment of HM, about $15 \%$ postmolar GTN and 4\% metastasis GTN occurred (Berkowitz and Goldstein, 2009).

Incidence of $\mathrm{HM}$ is different in various regions ranging from 0.6 to 4.27 per 1,000 pregnancies, while choriocarcinoma ranges from 1 to 9.2 per 40,000 pregnancies. However, incidence rates for HM in Europe and Asia has significantly declined due to improvement in medical technology, therapeutic agents and socioeconomic status (Lee et al., 2009).

In Thailand, incidence rates of GTD have been reported from several centers ranging from 1.67-4.27 per 1,000 deliveries (Sumawong, 1967; Benjapibal et al., 2000). Reported incidence of GTD from 1984-1995 at King Chulalongkorn Memorial Hospital (KCMH) showed an incidence of HM and GTD at 1 per 450 deliveries and 1 per 2,283 deliveries, respectively. About $14.6 \%$ of HM patients transformed into metastatic GTN, mostly to lung and vagina; and mainly treated with chemotherapy (Limpongsanurak, 1994; Limpongsanurak and Sitthisomwong, 1999).

In 2002, full implementation of the universal coverage policy in Thailand has changed pattern of patients' distribution from large, tertiary hospitals to smaller hospitals as the primary healthcare option. According to the policy, registered patients have to go to primary 
health care providers at the first place. As a result, an improvement in the availability and distribution of health workers, their skills and competencies in rural areas was observed. Thai people were able to access adequate health services from local area hospitals without incurring any medical fees (Tangcharoensathien et al., 2013). However, this correlation between changes in GTD and those made in the Thai healthcare system have not been reported.

The primary objective of this study is to find the incidence of hydatidiform mole in $\mathrm{KCMH}$ from 1994 to 2013 , comparing before and after universal coverage policy implementation. The secondary objective is to identify the changing of clinical profiles and patients' status over two decade.

\section{Materials and Methods}

After gaining approval from the Institutional Review Board of Research Affairs, Faculty of Medicine, Chulalongkorn University, NO. 92/2014, all women with clinical or pathological diagnosed of hydatidiform mole, gestational trophoblastic disease and choriocarcinoma in KCMH from January 1, 1994 to December 31, 2013 were retrospectively reviewed. Patient data was collected from inpatient databases including The International Classification of Diseases (ICD), 10th edition including Hydatidiform mole (O01), Gestational trophoblastic disease (D392), Choriocarcinoma (C58) and the KCMH outpatient database. Patients with loss of data records, wrong coding and wrong diagnosis confirmed by pathological reports were excluded from the study.

Patients were classified into three groups, HM, postmolar GTN and nonmolar GTN. General data comprised of, age, gravidity, parity, obstetric history, gestational age, status of health care coverage and referral status were collected. Clinical data of included clinical presentation, pretreatment serum $\mathrm{B}-\mathrm{hCG}$, hemoglobin (normal was defined as hemoglobin $(\mathrm{Hb})>11 \mathrm{~g} / \mathrm{dl}$, mild anemia was defined as $\mathrm{Hb} 10-10.9 \mathrm{~g} / \mathrm{dl}$, moderate anemia was defined as $\mathrm{Hb} 7-9.9 \mathrm{~g} / \mathrm{dl}$ and severe anemia was defined as $\mathrm{Hb}<7 \mathrm{~g} / \mathrm{dl}$ )(WHO, 2011), thyroid function test (normal range of free T3 2.4-4.2 pg/mL, free T4 0.8-1.7 $\mathrm{ng} / \mathrm{dL}$ and TSH 0.3-4.2 mIU (Cunningham et al., 2014)), subclinical hyperthyroid $(\mathrm{TSH}<0.1 \mathrm{IU} / \mathrm{L}$ with normal free
T3 and free T4), Massive blood loss defined as >1000 $\mathrm{ml}$, imaging studies, treatment and pathological report. According to criteria of the International Federation of Gynecology and Obstetrics (FIGO), 2002, postmolar GTN was defined as patients with post treatment HM with at least a following 1) B-hCG plateau for 4 consecutive values over 3 weeks, 2) B-hCG rise of $10 \%$ for 3 values over 2 weeks, 3) B-hCG persistence for 6 months after molar evacuation, 4) histopathologic diagnosis of choriocarcinoma, and 5) evidence of metastasis (Ngan et al., 2003).

As the universal coverage policy was initiated in 2002, data was divided into two groups according to a time period spanning over 2 decades. The first decade was from January 1, 1994 to December 31, 2003 and second decade from January 1, 2004 to December 31, 2013.

Overall incidence of GTD was calculated. Incidence of disease, patient characteristics, clinical presentation, treatment and remission of disease was compared between first and second decades, thereby representing the changes of disease before and after the implementation of universal coverage health system in Thailand.

\section{Statistical analysis}

Patient characteristics were analyzed by descriptive statistics and reported as frequencies, percentage, mean or median as appropriate. Chi-square test was used for the comparison of patient characteristics between each decade. Continuous data were analyzed by Mann-Whitney $\mathrm{U}$ test. A p-value $<0.05$ was considered statistically significant. All statistical analyses were performed using the Statistical Package for Social Sciences (SPSS) versions 17 for Windows (SPSS, Chicago, IL, USA).

\section{Results}

From 1994-2013, there were 479 cases of GTD. Data was collected from $435(90.8 \%)$ patients. There were $315(72.4 \%)$ of HM and $120(27.6 \%)$ of GTN at the first diagnosis. There were 204 and 111 cases of HM in the first and second decade respectively. Average delivery cases in first and second decades were 11,682 cases per year and 6,352 cases per year respectively. Overall incidence of $\mathrm{HM}$ was 1.70 per 1,000 deliveries. Incidence of HM

Table 1. Number of gestational trophoblastic disease cases according to group of disease and referral status at King Chulalongkorn Memorial Hospital, Bangkok, 1994-2013

\begin{tabular}{|c|c|c|c|}
\hline Groups of GTD $+\uparrow$ & $\begin{array}{c}1994-2003(\mathrm{n}=275) \\
\text { No. }(\%)\end{array}$ & $\begin{array}{c}2004-2013(n=160) \\
\text { No. }(\%)\end{array}$ & p-value \\
\hline $\mathrm{HM} \dagger$ & & & 0.27 \\
\hline Primary case & $125(47.6)$ & $60(38.1)$ & \\
\hline Referred case & $29(12.7)$ & $22(14.4)$ & \\
\hline Post molar GTN & & & 0.91 \\
\hline Following HM (primary case) & $31(11.3)$ & $17(10.6)$ & \\
\hline Following HM (referred case) & $10(3.6)$ & $7(4.4)$ & \\
\hline Following HM form other hospitals & $41(14.9)$ & $24(15)$ & \\
\hline Non molar GTN & & & $<0.01$ \\
\hline Primary case & $15(5.5)$ & $5(3.1)$ & \\
\hline Referred case & $12(4.4)$ & $23(14.4)$ & \\
\hline
\end{tabular}

$\dagger$ HM included only cases with disease remission;†† Missing value 14 (3.2\%) 
Epidemiology of Hydatidiform Moles in a Tertiary Hospital in Thailand over Two Decades: Impact of National Health Policy

in the first and second decades were $1.70(204 / 116,819)$ and $1.71(111 / 63,515)$ per 1,000 deliveries, respectively $(\mathrm{p}=0.65,95 \% \mathrm{CI} 1.54-1.88)$. Comparing between 19942003 and 2004-2013, cases of GTD first diagnosed at $\mathrm{KCMH}$ decreased significantly from 177 (67.3\%) to 86 $(54.4 \%, \mathrm{p}=0.01)$. The number of primary cases of HM were reduced from $125(47.5 \%)$ to $60(38 \%)$. In the group of referred cases, number of patients of HM, postmolar GTN and nonmolar GTN were 51(32.3\%), 72(45.6\%) and $35(22.2 \%)$, respectively. There were $67.7 \%$ of GTN cases referred from other hospitals. Referred cases of nonmolar GTN increased from $12(4.4 \%)$ in the first decade to 23
$(14.4 \%)$ in the second decade $(\mathrm{p}<0.01)$. Patients were categorized according to group of diseases and type of referral cases as shown in Table 1.

In 1994-2003, prior to the full implementation of the universal coverage healthcare system, most of medical fees were paid independently by patients in form of cash payment $(147,71 \%)$. However, the period after universal coverage implementation showed a significant increase in patient utilization of both universal health coverage and social security policies $(\mathrm{p}<0.01$ and $\mathrm{p}=0.02)$ to help towards the payment of incurred medical expenses. As a result of more government health and social policies, cash

Table 2. Distribution of gestational trophoblastic disease case by health security system at King Chulalongkorn Memorial Hospital, Bangkok, 1994-2013

\begin{tabular}{lccr}
\hline Health security system & \multicolumn{2}{c}{ No. of patients (n) } & p value \\
& 1994-2003 (n=207) & 2004-2013 (n=144) & \\
\hline Civil Servant Medical Benefit Scheme (CSMBS) & $22(10.6 \%)$ & $11(7.6 \%)$ & 0.67 \\
Social Security Scheme (SSS) & $21(10.1 \%)$ & $28(19.4 \%)$ & 0.02 \\
Universal Health Coverage (UC) & $17(8.2 \%)$ & $61(42.4 \%)$ & $<0.01$ \\
Cash payment & $147(71 \%)$ & $44(30.6 \%)$ & $<0.01$ \\
\hline
\end{tabular}

Missing value 84 (19.3\%)

Table 3. Patients and clinical characteristic of hydatidiform mole at King Chulalongkorn Memorial Hospital, Bangkok, 1994-2013

\begin{tabular}{|c|c|c|c|}
\hline \multirow[t]{2}{*}{ Patient characteristics } & \multicolumn{2}{|c|}{ No. of patients $(\%)$} & \multirow[b]{2}{*}{ p-value } \\
\hline & $1994-2003(n=207)$ & 2004-2013 (n=108) & \\
\hline \multicolumn{4}{|l|}{ Patients Characteristics (median) } \\
\hline Age (years) & 26 & 28 & 0.15 \\
\hline Gravida (n) & 2 & 2 & 0.59 \\
\hline Parity (n) & 0 & 0 & 0.85 \\
\hline Gestational age at diagnosis (weeks) & 13 & 13 & 0.88 \\
\hline ß-hCG $(\mathrm{IU} / \mathrm{ml})$ & 162,477 & 276,942 & 0.02 \\
\hline \multicolumn{4}{|l|}{ Pathological finding $\dagger$} \\
\hline Complete HM & $59(33.9 \%)$ & $81(77.9 \%)$ & \\
\hline Partial HM & $13(7.5 \%)$ & $17(16.3 \%)$ & \\
\hline HM, not categorized to complete or partial HM & $102(58.6 \%)$ & $6(5.8 \%)$ & \\
\hline \multicolumn{4}{|l|}{ Clinical presentations } \\
\hline Vaginal bleeding & $169(89.4)$ & $86(79.6)$ & 0.02 \\
\hline Enlarged uterine size & $33(17.5)$ & $29(26.9)$ & 0.06 \\
\hline Hyperemesis gravidarum & $44(23.3)$ & $21(19.4)$ & 0.44 \\
\hline \multicolumn{4}{|l|}{ Pregnancy-induced hypertension } \\
\hline Without severe feature & $5(2.6)$ & $2(1.9)$ & 0.33 \\
\hline With severe feature & $7(3.7)$ & $1(0.9)$ & \\
\hline Clinical Thyrotoxicosis & $13(6.9)$ & $3(2.8)$ & 0.13 \\
\hline Theca lutein cyst $>6 \mathrm{~cm}$ & $22(13.2)$ & $11(11.8)$ & 0.76 \\
\hline Asymptomatic & $9(4.8)$ & $11(10.2)$ & 0.07 \\
\hline Anemia at presentations $\uparrow \uparrow$ & & & 0.03 \\
\hline No & $73(44.2)$ & $60(68.2)$ & \\
\hline Mild anemia & $46(27.9)$ & $12(13.6)$ & \\
\hline Moderate anemia & $42(25.5)$ & $15(17.0)$ & \\
\hline Severe anemia & $4(2.4)$ & $1(1.1)$ & \\
\hline Thyroid function test & & & 0.02 \\
\hline Normal & $35(49.3)$ & $28(35.0)$ & \\
\hline Hyperthyroidism & $33(46.5)$ & $36(45.0)$ & \\
\hline Subclinical hyperthyroid & $2(2.8)$ & $14(17.5)$ & \\
\hline Subclinical hypothyroid & $1(1.4)$ & $2(2.5)$ & \\
\hline \multicolumn{4}{|l|}{ Remission rate $\dagger+\dagger$} \\
\hline Remission & $113(73.9)$ & $69(74.2)$ & 0.95 \\
\hline Post molar GTN & $40(26.1)$ & $24(25.8)$ & \\
\hline
\end{tabular}

$\dagger$ Missing value 37 (11.7\%); $\uparrow$ Missing value 62 (19.7\%); $\ddagger$ Missing value 164 (52.1\%); $\dagger \dagger$ Missing value $69(21.9 \%)$ 
payments also decreased significantly from $71 \%$ to $30.6 \%$ $(\mathrm{p}<0.01)$. The use of healthcare systems of GTD patients are shown in Table 2.

Among 315 cases of HM, median age was 26 years old (range, 14-53). Cases with aged less than 19 and over 40 years were $56(17.8 \%)$ and $32(10.2 \%)$, respectively. Median number of gravida and parity were 2 (range, 1-8) and 0 (range, 0-7), respectively. Median gestational age at diagnosis was 13 weeks (range, 2-37). There were no significantly different patient characteristics between the two decade periods in age, gravida, parity and gestational age as shown in Table 3 .

According to clinical presentation of HM, from first to second decade, vaginal bleeding was significantly decreased from $89.4 \%$ to $79.6 \%(\mathrm{p}=0.02)$. Asymptomatic patients increased from $4.8 \%$ to $10.2 \%(\mathrm{p}=0.07)$. Even though vaginal bleeding was the most common presenting symptom, number of patients with normal hemoglobin increased significantly in second decade from $44.2 \%$ to $68.2 \%(\mathrm{p}=0.03)$ and patients with mild anemia decreased from $27.9 \%$ to $13.6 \%$. Although, clinical thyrotoxicosis was not different and was found only in a small number of patients, subclinical hyperthyroid increased from $2.8 \%$ to $17.5 \%$ as demonstrated in table 3 .

The primary treatment of HM in both decades was suction \& curettage ( $83.4 \%$ and $79.4 \%) \quad(p=0.39)$, respectively. Massive blood loss was the major complication which significantly declined from $14 \%$ in the first decade to $6.5 \%$ in the second decade $(\mathrm{p}=0.04)$. Other complications were post-operative metritis (1.9\%), twisted/leakage of theca lutein cyst (1.3\%), pulmonary edema $(1.0 \%)$, pulmonary embolism $(0.3 \%)$, atrial fibrillation $(0.3 \%)$ and post-operative urinary retention $(0.3 \%)$.

After treatment, there were $69(21.9 \%)$ patients loss to follow up. The remaining number of cases in both decades were similar in remission rate $(n=113,73.9 \%$ and $69,74.2 \%$, respectively) and rate of postmolar GTN $(40,26.1 \%$ and $24,25.8 \%$, respectively) $(\mathrm{p}=0.95)$. In comparison, the number of postmolar GTN referred cases $(17,30.4 \%)$ was more than primary cases $(47,26.3 \%)$ $(\mathrm{p}=0.55)$.

\section{Discussion}

This study was conducted in King Chulalongkorn Memorial Hospital, a tertiary care hospital in Bangkok Thailand. From the study period, incidence of HM over two decades was 1.70 per 1,000 deliveries. Comparing to study from the same institute in 19841993 of Limpongsanurak et al., the incidence of HM in $\mathrm{KCMH}$ decreased from 2.22 to 1.70 per 1,000 deliveries (Limpongsanurak, 1994). Although the number of HM in this study was reduced by half in the second decade, the incidence between first and second decades reflected no change. This can be explained by decreasing number of delivered cases in tertiary hospitals over 20 years. The average delivery cases in first decade was 11,682 cases per year but decreased to 6,352 cases per year in the second decade. According to the universal coverage health policy, patients initially have to see primary doctors at registered hospitals and would be referred if the primary hospital cannot manage the disease. Therefore, most pregnant and uncomplicated HM patients were treated in primary hospitals by using their healthcare and social security benefits without incurring medical expenses. Only complicated cases were referred to tertiary care hospitals (Tangcharoensathien et al., 2013). Since GTN is more complicated than hydatidiform mole and needs specialized gynecologic oncologists and treatment facilities. In this study, GTN cases made up the majority of referred cases. We also noticed an increase in referral of nonmolar GTN cases in the latter decade. After implementation of the universal coverage health policy, referred patients with universal coverage health was $29.1 \%$ while independent cash payments decreased from $71 \%$ to $30.6 \%$.

The incidence of HM in this study was consistent with the studies from other major university hospitals in Thailand (Benjapibal et al., 2000; Lertkhachonsuk et al., 2012) and in other Asian countries (Kim et al., 1998; Matsui et al., 2003; Ozalp and Oge, 2013) but higher than European countries which was 0.57-1.1 per 1,000 pregnancies (Savage et al., 2010; Lybol et al., 2011). According to the previous studies, ethnics have an influence on incidence of HM. Asian populations are twofold more likely to develop HM than Caucasians. (Tham et al., 2003; Lybol et al., 2011).

Median age of HM and GTN were 26 and 30 years old, respectively, which can be explained by the high fertility rate of this age group. Other studies showed similar patient age groups (Limpongsanurak, 1994; Soto-Wright et al., 1995; Benjapibal et al., 2000; Hou et al., 2008; Lertkhachonsuk et al., 2012; Ozalp et al., 2014). HM can be diagnosed at early gestational age and asymptomatic patients were increased because of early detection of the disease by ultrasonography during antenatal care screening. However, unlike the previous studies (Ozalp and Oge, 2013), timing of diagnosis in this study did not change over two decades because it was depend on the time of first visit at antenatal clinic and first-trimester screening ultrasound did not applied to all pregnant women in our institute. The most common presentation of $\mathrm{HM}$ is vaginal bleeding. This presentation was reduced significantly in the second decade. Severity of anemia also improved when comparing between the two decades indicating that patients were able to access healthcare services and early treatment. Hyperthyroidism did not differ between the two decades but there was an increase in number of patients with subclinical hyperthyroidism. Unlike previous studies, asymptomatic patients of HM in our hospital increased over the 20 year study period but was not significant (Hou et al., 2008; Mangili et al., 2008; Lertkhachonsuk et al., 2012).

Postmolar GTN after treatment of HM in our hospital was about $26 \%$. Both primary and referred cases had similar GTN rates. This is consistent with the study of Lertkhachonsuk et al. (2012) but higher than others (SotoWright et al., 1995; Benjapibal et al., 2000; Hou et al., 2008). As a referral center, more complicated and higher risk of complete HM cases may effect postmolar GTN rates in this study. As we know from previous studies, early diagnosis did not prevent postmolar GTN. Therefore, close 
Epidemiology of Hydatidiform Moles in a Tertiary Hospital in Thailand over Two Decades: Impact of National Health Policy

follow up of patients after treatment is necessary (SotoWright et al., 1995; Hou et al., 2008; Lertkhachonsuk et al., 2012; Ozalp and Oge, 2013).

There were a few study limitations worth mentioning. Due to the nature of a retrospective study, a large portion of our data was lost as archived or inactive patient data is expunged from the hospital database system after five years. Rate of loss to follow up patients was high in HM which obstructed our ability to define the long term remission rate. Some cases were not histological categorized into complete or partial HM, therefore the exact ratio of complete HM to partial HM cannot established.

In conclusion, the number of HM cases in this study decreased over 2 decades but incidence was unchanged. Total numbers of HM and delivery rates decreased as a result of distributions of disease to primary hospitals, a direct impact of the national health policy implementation. Referral rates of GTN also increased due to limitation of treatment capacity in primary hospitals. Classic clinical presentation was decreased significantly in the last decade.

\section{Acknowledgements}

This study has no financial grant supported.

\section{References}

Benjapibal M, Boriboonhirunsarn D, Suphanit I, et al (2000). Molar pregnancy: clinical analysis of 151 patients. Thai $J$ Obst Gynaecol, 12, 35-41.

Berkowitz RS, Goldstein DP (2009). Current management of gestational trophoblastic diseases. Gynecol Oncol, 112, 654-62.

Berkowitz RS, Goldstein DP (2012). Gestational trophoblastic disease. In 'Berek\& Novak's gynecology', Eds Lippincott Williams\&Wilkins, California,

Cunningham FG, Leveno KJ, Bloom SL, et al (2014). Appendix. In 'Williams Obstetrics', Eds McGraw-Hill Education, The United states, 1290

Hou J, Wan X, Xiang Y, et al (2008). Changes of clinical feathers in hydatidiform mole analysis of 113 cases. J Reproduct Med, 53, 629-33.

Kim SJ, Bae SN, Kim JH, et al (1998). Epidemiology and time trends of gestational trophoblastic disease in Korea. Int $J$ Gynecol Obstet, 1, 33-8.

Lee C, Smith HO, Kim SJ (2009). Epidemiology In 'Gestational trophoblastic disease', Eds Sheffield,

Lertkhachonsuk A, Israngura N, Tangtrakul S, et al (2012). Complete hydatidiform mole change in clinical profile over three decades. J Reproductive Med, 57, 470-4.

Limpongsanurak S (1994). An epidemiological study of hydatidiform moles conducted in Chulalongkorn Hospital. Chulalongkorn Med J, 38, 659-65.

Limpongsanurak S, Sitthisomwong T (1999). Metastatic gestational trophoblastic neoplasia at King Chulalongkorn Memorial hospital. Trophoblast Res, 13, 205-11.

Lurain JR, Brewer JI (2010). Gestational trophoblastic disease I: epidemiology, pathology, clinical presentation and diagnosis of gestational trophoblastic disease, and management of hydatidiform mole. Am J Obstet Gynecol, 203, 153-9.

Lybol C, Thomas MG, Bulten J, et al (2011). Increase in the incidence of gestational trophoblastic disease in The Netherlands. Gynecol Oncol, 121, 334-8.
Mangili G, Garavaglia E, Cavoretto P, et al (2008). Clinical presentation of hydatidiform mole in northern Italy: has it changed in the last twenty years? Am J Obstet Gynecol, 198, 1-4.

Matsui H, Iitsuka Y, Yamazawa K, et al (2003). Changes in the incidence of molar pregnancies. A population-based study in Chiba prefecture and Japan between 1974 and 2000. Human Reproduct, 18, 172-5.

Ngan HY, Kohorn EI, Cole LA, et al (2012). Trophoblastic disease. Int J Gynaecol Obstet, 119, 130-6.

Ngan HYS, Bender H, Benedet JL, et al (2003). Gestational trophoblastic neoplasia, FIGO staging and classification. Int J Gynecol Obstet, 83, 175-7.

Ozalp SS, Oge T (2013). Gestational trophoblastic diseases in Turkey. J Reprod Med, 58, 67-71.

Ozalp SS, Telli E, Oge T, et al (2014). Multicenter analysis of gestational trophoblastic neoplasia in Turkey. Asian Pac J Cancer Prev, 15, 3625-8.

Savage P, Williams J, Wong SL, et al (2010). The demographics of molar pregnancies in England and Wales from 2000-2009. $J$ Reproduct Med, 55, 341-5.

Soto-Wright V, Bernstein M, Goldstein DP, et al (1995). The change of clinical presentation of complete molar pregnancy. Obstet Gynecol, 86, 775-9.

Sumawong V (1967). Hydatidiform mole and its treatment in Chiengmai, Thailand. J Med Associat Thailand, 50, 1-13.

Tangcharoensathien V, Limwattananon S, Suphanchaimat R, et al (2013). Health workforce contributions to health system development: a platform for universal health coverage. Bulletin World Health Organizat, 91, 874-80.

Tham BW, Everard JE, Tidy JA, et al (2003). Gestational trophoblastic disease in the Asian population of Northern England and North Wales. BJOG Int J Obstet Gynaecol, 110, 555-9. 